\title{
Laser Photography Device - Spatial Parameters of Imaging
}

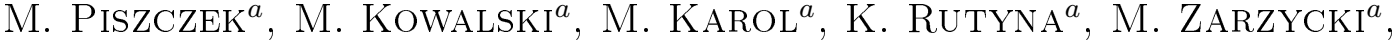 \\ M. SZUSTAKOWSKI ${ }^{a}$ AND K. LUDWIKOWSKI ${ }^{b}$ \\ ${ }^{a}$ Institute of Optoelectronics, Military University of Technology, S. Kaliskiego 2, 00-908 Warsaw, Poland \\ ${ }^{b}$ HARDsoft Microprocessor Systems, Cegielniana 4A, 30-404 Kraków, Poland
}

Laser photography device is an imaging device developed in the Institute of Optoelectronics, Military University of Technology and it is an example of modern image acquisition device. The laser photography device allows to define a $3 \mathrm{D}$ observation scene thanks to short-time scene illumination and image acquisition method. This device works according to time-spatial framing method. In the paper, basics of time-spatial framing method are explained. Special attention is given to time parameters of device and their influence to spatial parameters of registered images. In this paper the laser photography device and results of chosen experiments are presented and described. Experimental results presented in the paper show the potential and possibilities of using the laser photography device as a camera for observation and measurement applications.

DOI: $10.12693 /$ APhysPolA.124.550

PACS: 42.30.Sy, 42.30.Tz, 02.60.-x

\section{Introduction}

Modern vision systems offer great observationmeasurement possibilities and play huge role in the recent information systems. One of the most interesting vision devices available on the market are time-of-flight (ToF) cameras [1,2]. A characteristic feature of these active imaging devices is the ability to measure distances to objects. We can distinguish several types of ToF cameras according to the method of acquisition of spatial information. One of the methods is range gated imaging, which offers the ability to selectively register imaging information. Special property of the range gated imaging consists of the possibility to register image for defined distance and depth of observation [3,4]. Registered images are not just a projection of angular observation range but are a representation of precisely defined three-dimensional fragment of space.

The laser photography device (LPD) developed in the Institute of Optoelectronics, Military University of Technology operates according to the time-spatial framing method [5]. This method is one of the most sophisticated solution amongst imaging techniques. The range of observation of the LPD is strictly connected with the conditions of propagation of electromagnetic wave. When operating in standard atmospheric conditions with using high-energy nanosecond laser pulses and nanosecond shutter, it is possible to register images on distances below $1 \mathrm{~km}$ with the spatial resolution below $1.5 \mathrm{~m}$.

\section{Method characteristics}

The key elements of most of recently used imaging technologies are the characteristic parameters of method. These parameters are mainly responsible for the observation-measurement capabilities and potential applications of method. The characteristic parameters of the time-spatial framing method are: a method of scene illumination (short-time laser pulses $T_{\mathrm{i}} \approx 10^{-8} \mathrm{~s}$ ) and a method of light detection - fast shutter $T_{\mathrm{d}} \approx 10^{-8} \mathrm{~s}$. The most important aspect of this method is the proper settings of time relations between emitted and detected electromagnetic wave propagating in the measurement space with the speed value $c \approx 3 \times 10^{8} \mathrm{~m} / \mathrm{s}$. According to the basic equation $R=c T$ connecting the path of wave (laser pulse) $R$ with time $T$, there is a possibility to register imaging information from the precisely defined fragment of space and time interval [6].

The fact of using the laser radiation, and the accurate relationship between time and space of imaging, was the reason of naming the method time-spatial framing method and laser photography.

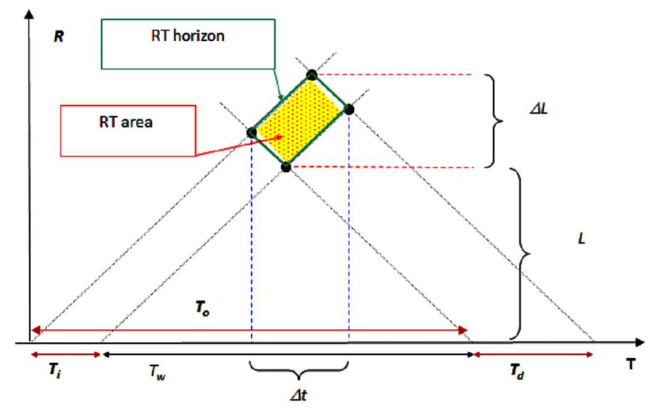

Fig. 1. $\quad R-T$ diagram.

For better understanding, the time-spatial framing method in the $R-T$ diagram was proposed (Fig. 1). This diagram connects the information of time and space of imaging in a $2 \mathrm{D}$ graph.

Basic dependences:

- Time period of registered events $\Delta t=0.5\left(T_{\mathrm{w}}+T_{\mathrm{i}}\right)$;

- Distance to observation area $l_{\min }=0.5 c T_{\mathrm{w}}$;

- Depth of observation area $\Delta L=0.5 c\left(T_{\mathrm{d}}+T_{\mathrm{i}}\right)$. 
Because of higher precision and easier hardware implementation, the $T_{\mathrm{w}}$ parameter (waiting time) was replaced with a $T_{0}$ parameter (delay time).

$$
T_{0}=T_{\mathrm{w}}+T_{\mathrm{i}} .
$$

Based on above equations, one can conclude that definition of distance and depth of observation area can be realized in many different ways depending on times $T_{\mathrm{i}}$, $T_{0}$ and $T_{\mathrm{d}}$.

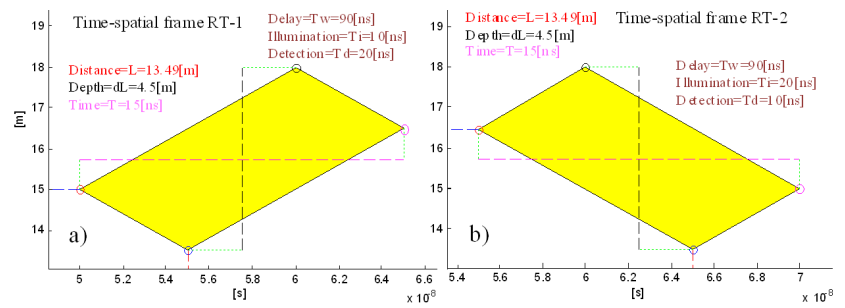

Fig. 2. Examples of time-spatial frames.

Examples of time-spatial frames in various configurations $T_{\mathrm{i}}<T_{\mathrm{d}}$ and $T_{\mathrm{i}}>T_{\mathrm{d}}$ are presented in Fig. 2. Apparently, Fig. 2a and b presents the same time-spatial frame. In terms of space both figures are the same but the registration took place in different time intervals in reference to the beginning of time of illuminator pulse.

From a point of view of acquisition of spatial information it is irrelevant, but besides the time gap, these two acquisitions are different because of the energetic deficit effect in variant $T_{\mathrm{i}}>T_{\mathrm{d}}$.

\section{Device characteristics}

Theoretical analysis helped us to define the key components of the camera (Fig. 3) using the time-spatial framing method:

a) laser illuminator,

b) image acquisition module,

c) time parameters driver,

d) camera controller.

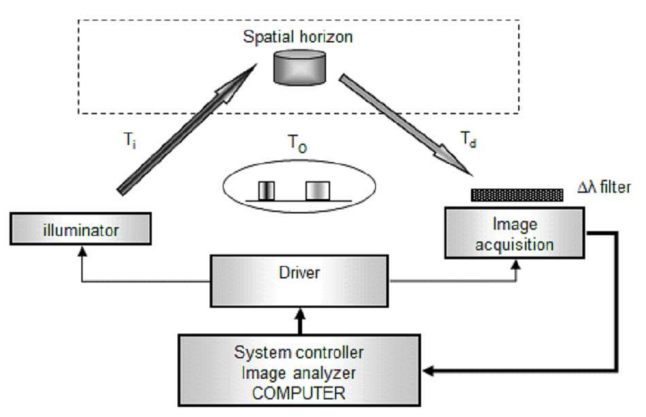

Fig. 3. Key camera components.

\subsection{Illuminator}

The laser photography device requires a pulse illuminator with parameters defined by the time-spatial framing method. The key illuminator parameters are short pulse duration time (few nanoseconds) and high energy light pulse [7]. The bigger the laser pulse energy is, the bigger distances and objects of bigger angular sizes the LPD can register.

The LPD can operate in two configurations - mobile and stationary. A mobile version is dedicated to work in shorter distances (potential future applications - handheld observation equipment e.g. binocular, gunsight or rocket system target illuminator) and uses semiconductor lasers.

The device is equipped with two semiconductor lasers with adjustable pulse duration (pulse laser diodes). Parameters of semiconductor lasers are listed in Table I.

Parameters of semiconductor lasers.

TABLE I

\begin{tabular}{c|c|c}
\hline \hline Wavelength & $850 \mathrm{~nm}$ & $905 \mathrm{~nm}$ \\
\hline power & $100 \mathrm{~W}$ & $220 \mathrm{~W}$ \\
pulse duration & $15-30 \mathrm{~ns}$ & $30-100 \mathrm{~ns}$ \\
max. trigger repetition & $20 \mathrm{kHz}$ & $5 \mathrm{kHz}$ \\
\hline & & \\
& &
\end{tabular}

In stationary version, dedicated for long-distance operating, high-energy laser is used. This laser offers a maximum energy of $360 \mathrm{~mJ}$ and it is operating at the second harmonic $(532 \mathrm{~nm})$ with nanosecond pulse durations (3-6 ns) and a TOP-HAT beam profile. Parameters of laser illuminators are controllable according to the requirements of the time-spatial framing method.

\subsection{Image acquisition module}

One of the most important elements of the LPD is a MCP (micro-channel plate) [8]. Thanks to this element it is possible to open a shutter for a very short time $\approx 3$ ns. Another very important properties of the MCP is high sensitivity, amplification of image $\left(10000 \mathrm{~cd} / \mathrm{m}^{2} / \mathrm{lx}\right)$ and extension of image glossing time. Image is registered by a synchronous camera module equipped with CCD ProgressiveScan sensor with the resolution of $1360 \times 1024$ pixels.

\subsection{Driver module}

The image acquisition method of the LPD requires to use a driver module for synchronization of modules of illuminator and image acquisition. A prototype driver module can generate controlling signals with the resolution of $1 \mathrm{~ns}$ with jitter $<0.2 \mathrm{~ns}$.

All of elements of the system - the MCP, the camera, a controlling module, the semiconductor laser illuminator and a PC-computer are mounted in one housing. This 
means that the system is compact and mobile. It is expected to use a battery power supply. The block diagram of the LPD is presented in Fig. 4.

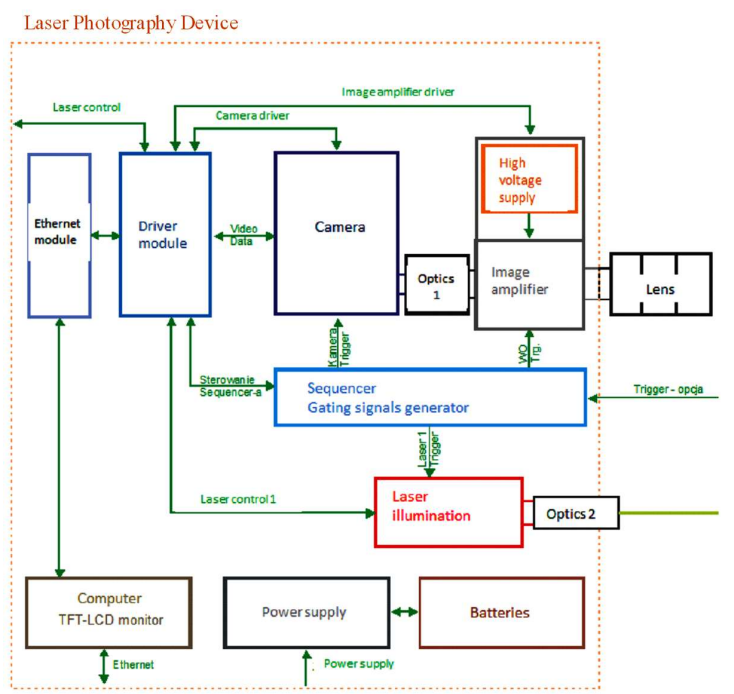

Fig. 4. The block diagram of the LPD.

On the right side of the front panel, the two semiconductor laser illuminators were mounted. On the left side of the front panel of the LPD, the mounting bracket for $F$-mount lenses was placed. On the rear panel, all the required connectors for connecting external devices (power supply, control and communication cables of the LPD) were mounted.

A housing of the laser photography device was made of powder painted duraluminum. Internal construction (chassis) allows user a simple assembly/disassembly of all components of the LPD. External elements hide assembly elements and screws. This design of housing guarantees the proper mechanical resistance and drop strength. Laser photography device can cooperate with external illuminators.

\section{Device tests}

Experimental laser photography device developed in the IOE MUT is an observation-measurement device. As every vision device, the LPD can be described using a set of parameters. We can distinguish:

- standard properties - typical for classic imaging devices,

- unique properties — characteristic only for the LPD.

To determine the values of parameters the following tests were run:

a) Investigations of transmission modules in case of time, spatial, spectral and radiometric parameters;

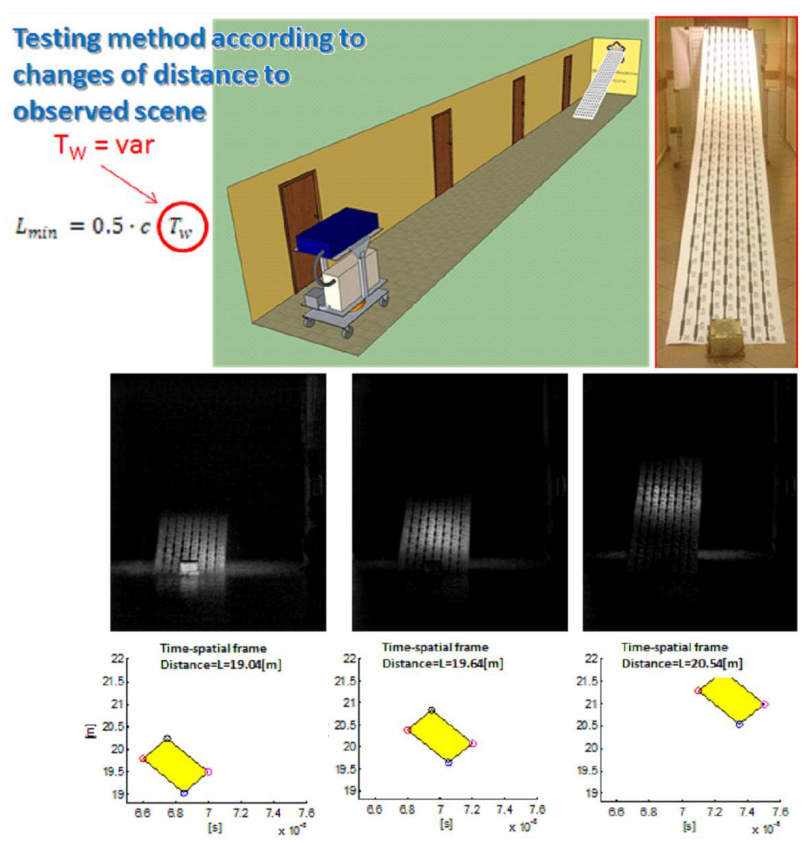

Fig. 5. Testing method according to changes of distance to observed scene.

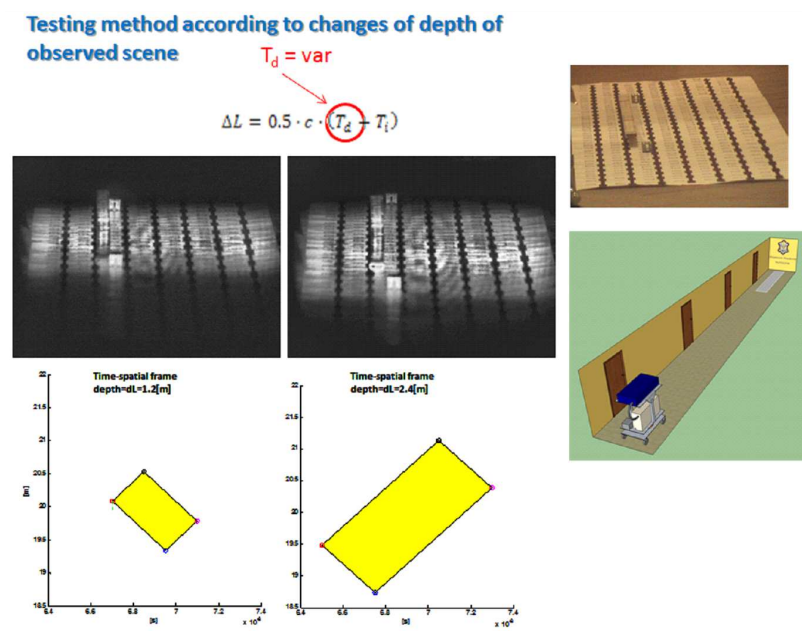

Fig. 6. Testing method according to changes of depth to observed scene.

b) Set of sequencer tests:

- sequencer software tests - correctness of time parameters of LPD modules and time instability of generated time sequences (jitter),

- generating time sequences for scanning of observed scene,

— tests for determining:

- minimal growth of observation distance,

- minimal depth of observation.

Most of the tests were run with arranged measurement scenes in laboratory conditions. Examples of the most 
essential LPD verification tests are shown in Fig. 5 and Fig. 6. These tests were run to verify the time-spatial framing method used in the LPD.

Figure 5 presents how the extension of waiting time influences the displacement of spatial frame. Modification of detection (presented in Fig. 6) time shows how the spatial frame is expanding during the rise of detection time. All the test results confirm the possibility of modifying spatial parameters of registered frames by proper control of the image acquisition parameters.

\section{Summary}

The most important parameters of the laser photography device are presented in Table II.

The main LPD parameters.

TABLE II

\begin{tabular}{|c|c|c|c|}
\hline $\begin{array}{l}\text { Image resolution } \\
\text { MTF resolution }\end{array}$ & \multicolumn{3}{|c|}{$\begin{array}{c}1360(H) \times 1024(V) \text { pixels } \\
60 \mathrm{lp} / \mathrm{mm}\end{array}$} \\
\hline \multicolumn{4}{|c|}{ Field of view (FOV) } \\
\hline - for optics $105 \mathrm{~mm}$ & \multicolumn{3}{|c|}{$11.04^{\circ}$} \\
\hline — for optics $500 \mathrm{~mm}$ & \multicolumn{3}{|c|}{$2.46^{\circ}$} \\
\hline — for optics $2800 \mathrm{~mm}$ & \multicolumn{3}{|c|}{$0.38^{\circ}$} \\
\hline Bit resolution & \multicolumn{3}{|c|}{ 14-bit } \\
\hline \multicolumn{4}{|c|}{ Spatial resolution } \\
\hline — min. observation distance & $\begin{array}{c}16 \mathrm{~m}-\text { for } 532 \mathrm{~nm} \\
\text { illluminator }\end{array}$ & \multicolumn{2}{|c|}{$\begin{array}{c}0 \mathrm{~m} \text { - for } 850 / 905 \mathrm{~nm} \\
\text { illluminators }\end{array}$} \\
\hline - min. depth of observation & \multirow{4}{*}{\multicolumn{3}{|c|}{$\begin{array}{c}1.2 \mathrm{~m} \\
0.15 \mathrm{~m} \\
0.15 \mathrm{~m} \\
1.5 \mathrm{~cm}\end{array}$}} \\
\hline — minimal distance growth & & & \\
\hline — minimal depth growth & & & \\
\hline — spatial jitter & & & \\
\hline Spectra range of illuminators & \multicolumn{3}{|c|}{$850 \mathrm{~nm}$} \\
\hline Max. energy & $360 \mathrm{~mJ}$ & & \\
\hline Max. power & & $0 \mathrm{~W}$ & $200 \mathrm{~W}$ \\
\hline Pulse duration & $5 \mathrm{~ns}$ & $-30 \mathrm{~ns}$ & $8 \mathrm{~ns}$ \\
\hline
\end{tabular}

The experimental results confirmed the possibility of building the fully functional camera working with the time-spatial framing method. The results of experiments also proved the ability of device to register images of precisely defined fragment of three-dimensional space.

Future devices and optoelectronic systems using this method of acquisition of imaging information can find many applications as observation-measurement tools.

\section{Acknowledgments}

The device presented in this paper was developed during project funded by the Polish Ministry of Science and Higher Education (project OR00000312).

\section{References}

[1] S. Schuon, C. Theobalt, J. Davis, S. Thrun, in: IEEE Computer Society Conf. on Computer Vision and Pattern Recognition Workshops, Anchorage, Alaska, 2008.
[2] M. Piszczek, M. Kowalski, Laser Photography in Selective Space Imaging and Navigation, Springer, 2013, Ch. IV.

[3] P. Andersson, Opt. Eng. 45, 034301 (2006).

[4] A. Medina, F. Gayá, F. Pozo, J. Opt. Soc. Am. A 23, 800 (2006).

[5] A. Medina, Three Dimensional Camera and Rangefinder, January 1992, United States Patent 5081530.

[6] X. Shiwei, Z. Shengchong, T. Shuwei, in: 2011 Int. Conf. on Electronics and Optoelectronics (ICEOE 2011), Dalian, 2011.

[7] M. Piszczek, M. Kowalski, M. Szustakowski, K. Rutyna, K. Ludwikowski, Acta Phys. Pol. A 122, 862 (2012).

[8] M. Piszczek, K. Rutyna, M. Szustakowski, Europ. Phys. J., ST 154, 153 (2008). 